\title{
A Novel Method for Logistics Facility Location by Combining Finite State Automaton and Genetic Algorithm
}

\author{
Liang Wang* \\ Anhui Normal University \\ AHNU \\ Wuhu, Anhui, China \\ liangw_2008@163.com \\ Lei Gao \\ CSIRO Land and Water \\ Glen Osmond SA 5064, Australia \\ lei.gao@csiro.au
}

\author{
Wanggen Li \\ Anhui Normal University \\ AHNU \\ Wuhu, Anhui, China \\ xchen@mail.ahnu.edu.cn
}

\begin{abstract}
The location of distribution centers plays a vital role in logistics planning. This paper presents a new method for solving the location problems. The mechanism of finite state automaton is combined to the standard GA to diversify location strategies and accelerate the convergence speed of the method. The experiment results demonstrate the proposed method outperforms the standard GA in terms of computational cost.
\end{abstract}

Keywords-facility location; genetic algorithm; finite state automaton

\section{INTRODUCTION}

As crucial problems in logistics planning, how to select distribution centers has been studied for decades. Solutions of this type of location problems fall in three categories continuous models, discrete models [1]-[3], and expert consultation models [4]. Among them, discrete location models are more practical, compared with discrete models that may obtain meaningless results. These discrete models aim to find the best location in limited alternatives [5]-[7]. Since these alternative locations are deterministic, the obtained solutions are in line with actual requirements.

Automata theory was initially proposed in robot research fields for imitating the human's or the animal's actions and behaviors. Nowadays, it has become one of theory foundations of computer science and has been widely used in computer as well as other fields. Finite state automaton (FSA) is based on the mathematical model of automata. The FSA was proposed as a kind of calculation models and focuses on the calculation in a limited storage process with discrete inputs and outputs. It has finite states, each of which can be converted to one or more states by a certain condition, or can be converted to null state. Such a mechanism of the FSA can be used to describe the logistics facility location problem.

This paper combined FSA with genetic algorithm (GA) as a new method for selecting distribution centers. The new method offers an improved processing speed and a better interpretation of the logistics location process. Meanwhile, the two algorithms (FSA and GA) complement and cooperate each other, enhancing the robustness of the combined method.

\section{THE COMBINED METHOD}

\section{A. Genetic Algorithm}

GA was first proposed by Holland, professor of the University of Michigan in 1975 in his monograph "Adaptation in Natural and Artificial Systems" [8]. It is based on Darwin's theory of evolution, using the survival of the fittest concept to simulate the process of evolution in biology. This algorithm includes four steps: problem initialization, individual evaluation, population evolution, and test termination. In the process of this algorithm, candidate individuals evolve as a result of the operations of crossover and mutation, then excellent individuals retain in accordance with certain criteria. After that, these excellent individuals are combined to produce a new generation of potentially outstanding individuals. By repeating and iterating the above process, a set of optimal solutions can be finally selected.

\section{B. Finite State Automaton}

FSA [9] is the mathematical language that describes finite states and transformations between these states. Its working processes are: at any moment in a time series sequence, it reads a character from an input string, and then does some transformation based on the current state and the input character. After finishing these, it goes to the next subsequent moment [10].

FSA consists of a finite set of states, which can be described by a five element equation $[9,11,12]$, that is, $M=\left(Q, ?, d, q_{0}, F\right)$. In this equation:

1) $Q$ is the set of finite states, where each element is called a state of the automaton. 
2) $\sum$ is an alphabet, which is the set of all characters in the input tape.

3) $\delta$ is a state transformation function, that is $\delta(q, x)=q^{\prime}$. It means that the automaton will turn to state $q^{\prime}$ after receiving character $x$ in state $q$. It describes the process of automaton from one state to the next.

4) $q_{0} \in Q$ is the initial state.

5) $F \in Q$ is the set of receiving states.

\section{Combining FSA with GA}

The encoding of a deterministic finite state automaton can be expressed by two parts of state transformation and output. Suppose the automaton has states [13], thus its input matrix can be expressed as $I=\left[\begin{array}{llll}q_{1} & q_{2} & \mathrm{~L} & q_{n}\end{array}\right]$, where $q_{1}$ is the initial state of automaton. Based on this, the state transfer matrix can be expressed:

$$
T=\left[\begin{array}{cccc}
t_{11} & t_{12} & \mathrm{~L} & t_{1 n} \\
t_{21} & t_{22} & \mathrm{~L} & t_{2 n} \\
\mathrm{M} & \mathrm{M} & \mathrm{M} & \mathrm{M} \\
t_{n 1} & t_{n 2} & \mathrm{~L} & t_{n n}
\end{array}\right]
$$

Where $t_{i j}$ belongs to the finite alphabet and denotes the transformation from state $q_{i}$ to $q_{j}$, that is $q_{j}=\delta\left(q_{i}, t_{i j}\right)$. $t_{i j}=0$ means there is no transformation between state $q_{i}$ and $q_{j}$. The output matrix of this automaton can be written as $O=\left[o_{1}, o_{2}, \mathrm{~L}, o_{n}\right]$, where $o_{i}$ means the $i-t h$ output state of automaton. So in the $n$ output states of the automaton, any state can be represented by the elements of the output matrix $O$.

Before combining the FSA with the standard GA, we introduce two definitions below.

\section{Definition 1}

If there are $n$ " 1 "s in a $0-1$ matrix of $n \times n$, and these " 1 "s are located in different rows and columns of the matrix. Then the matrix is regarded as a standard matrix.

\section{Definition 2}

The element change of the matrix is called as an operation of matrix mutation.

Next, combining with GA, we can deal with the input matrix and state transformation matrix of the automaton. Both the matrices are random $0-1$ ones. We first executed the 'mutate' operation to the input matrix. An element in the input matrix is selected randomly to mutate and produce a new individual, and then a new input matrix is obtained.

The specific process of mutating the transfer matrix into a standard matrix is shown as follows:
1) Change the elements in row $i$ : if there is only one " 1 " in these elements and the elements in the column of this element are all " 0 "s, then keep the elements of position $(i, j)$ unchanged; if there are one or more " 1 " in the column of these elements, then mutate them into " 0 ".

2) If there are two or more " 1 "s in the elements of row $i$, and the corresponding elements of column $j$ are all " 0 "s: then retain " 1 " of any positions in these elements and mutate " 1 "s into " 0 "s of the other positions in row $i$. If there is only one other element of column $j$ is " 0 " in the all " 1 " elements of row $i$, then retain the position $(i, j)$ and mutate " 1 "s into " 0 "s of the other positions in row $i$. If there are two or more " 1 "s in the elements of row $i$, and at least one " 1 " exists in the corresponding elements of column $j$, then retain " 1 " of any positions in these elements and mutate " 1 " into " 0 " of this "1" position and the other position in row $i$.

3) If the elements of row $i$ are all " 0 " $s$, then take any column $j$ where all elements are " 0 "s, and mutate " 0 " into " 1 " in the position $(i, j)$.

Repeat the above steps (1)-(3) to mutate the state transformation matrix of the automaton into a standard matrix. Then by multiplying the standard state transformation matrix by the input matrix of the automaton, we can obtain an output matrix of the automaton. Thus using GA to mutate the state transformation matrix and the output matrix of the automaton, some new individuals are obtained by optimizing their fatherlevel individuals. The states of the automaton, which are produced by the above evolution process, are the final result.

\section{AN APPLICATION TO LOGISTICS FACILITY LOCATION}

The problem of locating distribution centers is related to the path relationships among factory, distribution centers and customers. The solution to this problem is to find alternative locations that minimize total transportation cost. There could be a few factors that affect the location of distribution centers. One needs to do a comprehensive analysis on these factors, considering the relationships among them and the corresponding cost, and determine the fitness function based on the analysis. We determined the input matrix and transformation matrix of the automaton, used GA to mutate them, and output the states of the automaton. In our case, there are $n$ alternative locations, the goal of this case study is to select multiple optimal locations from these $n$ alternative locations to build distribution centers. Thus this case study aims to find a set of optimal solutions rather than one.

\section{A. Problem Description}

The formation of a logistics center is an inevitable result of society development. The logistics center considered in this case study is the urban distribution center [14]. There are three key facilities in the case study: factories, distribution centers and customers. Among them, each factory has a fixed location; the locations of customers are dispersed in multiple fixed areas; there are multiple alternative locations available for building distribution centers. The goal of this problem is to find one or more optimal locations with the minimum cost. 


\section{B. Model Description}

Based on the description of the problem, model parameters are given as follows:

\section{$n$ : The number of alternative distribution centers}

$t$ : The number of customers in the case study city

\section{$l:$ The number of factories}

$a_{i j}$ : Transport 1 unit price from distribution center $S_{i}$ to user $U_{j}$ (Yuan per unit weight distance)

$a_{i k}$ : Transport 1 unit price from distribution center $S_{i}$ to factory $F_{k}$ (Yuan per unit weight distance)

$d_{i j}$ : Distance from $S_{i}$ to $U_{j}$

$d_{i k}:$ Distance from $S_{i}$ to $F_{k}$

$w_{i}$ : The distribution capacity of $S_{i}$

$w_{i j}$ : User $U_{j}$ demands for $S_{i}$

$v_{k}$ : The supply capacity of $F_{k}$

$v_{i k}: S_{i}$ demands for factory $F_{k}$

$c_{i}$ : Management and construction costs of $S_{i}$

$y_{i}: 0-1$ variable, which equals " 1 " when an alternative logistics center is selected; otherwise, equals " 0 "

$B$ : The total budget for the management and construction of distribution centers

\section{$z$ : The total cost of transportation and distribution}

According to the above symbols, this model aims to minimize the total distribution cost $\mathrm{z}$. The objective function is given below:

$$
\mathrm{Z}=\sum_{i=1}^{n}\left(\sum_{j=1}^{t} a_{i j} w_{i j} d_{i j}+\sum_{k=1}^{l} a_{i k} v_{i k} d_{i k}+y_{i} c_{i}\right)
$$

where the variables are all non-negative, and there are some constraints:

$$
\begin{array}{ll}
\sum_{i=1}^{n} y_{i} c_{i} \leq B & i=1, \ldots n \\
\sum_{j=1}^{t} w_{i j} \leq w_{i} & j=1, \ldots t \\
\sum_{i=1}^{n} v_{i k} \leq v_{k} & k=1, \ldots l
\end{array}
$$

The objective function is to find a minimal cost with the constraints of not exceeding the given budget and the supply demand. Due to the undetermined number of finally selected distribution centers, the average investment cost for a distribution center was used as the fitness function value.
Finally, we used the proposed combined model to solve the optimization problem.

\section{Experiments}

The case study is modified from the case in the literature [3]. Suppose the case study city has three factories in an area of 6400 square kilometers. Goods are transported from factories to distribution centers, and then the centers will distribute them to customers in need. Ten alternative locations are available for building one or more distribution centers. The case study takes place in a city where customers are scattered, so we divided them into customer blocks. Suppose 600 customer blocks are distributed in the city and the demands of these customer blocks are no more than 800 goods units. The unit transportation price is 1 Yuan (Chinese dollar) per kilogram per kilometer. The distribution capacity of each distribution center and the supply capacity of each factory are both 150000 . Customers' demands for a distribution center and a distribution center's demands for a factory are all proportional to the distance between them and the distribution center. The ratio is a random number from one to ten. The management and construction cost of each distribution center is 2 billion Yuan. The total investment budget is 20 million Yuan. The locations of the alternative centers, factories and customers are produced randomly in a certain area. The output matrix of the automaton represents these selected locations..

\section{Results and Analyses}

Fig. 1 demonstrates the locations of factories, customers and alternative distribution centers. In the figure, green dots represent factories, blue dots represent customers, and red dots represent alternative locations. The evolution of the fitness function is shown as Fig. 2. Compare Figs 2(a) and 2(b), we can see that our proposed algorithm can improve the convergence speed.

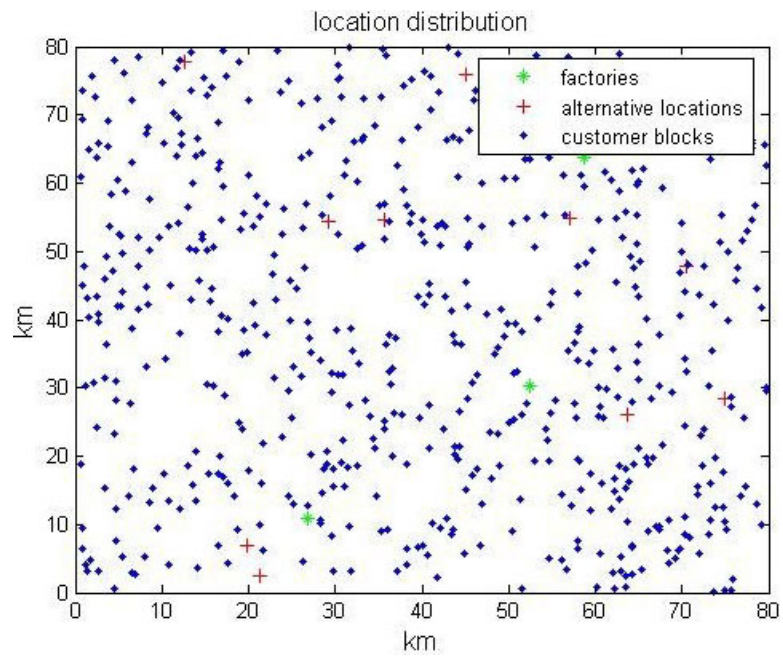

FIGURE I. THE LOCATION DISTRIBUTION OF FACTORIES, CUSTOMERS AND ALTERNATIVE LOCATIONS

Compared with the traditional location algorithm (standard GA) of the facility location, the algorithm proposed in this paper not only introduces the matrix encoding based on FSA to deal with the input state matrix and the transformation matrix, but also uses GA to mutate the encoding matrix. The proposed 
algorithm uses GA to deal with the matrix encoding of the automaton, ensures the diversity of individuals, and improves the convergence speed of the evolutionary curve, thus achieves an optimal effect. We can see from the graph of the evolutionary curve. Optimal solutions were found by our combined method with only 130 generations/iterations, while the standard GA needs 250 generations to find satisfactory results. When the two methods are applied to the facility location problem in a larger scale city, the benefit of our combined method will be much in evidence.

\section{CONCLUSIONS}

Aim to deal with the limitation of applying the standard GA in solving the facility location problem, we gave the selection process for the alternative locations through the state transformation of the FSA. Due to the difficulty in handling the illegal individuals that are produced in the encoding of the standard GA, the FSA-based matrix encoding was proposed in this paper. In addition, some operations of standard GA, such as mutation, were integrated to the method to obtain effective output states.

The method proposed in this paper can offer real-time and effective decisions to improve the operational efficiency of a logistics system. We shall make efforts in our future work to use ecosystem-inspired mechanisms [15]-[16] to improve the flexibility of the optimization method presented in this paper. The combined method can also be applied to broader areas, such as constrained mixed-variable optimization problems [17].

\section{ACKNOWLEDGMENT}

The authors gratefully acknowledge the financial support provided by the Project of Science and Technology of Wuhu in Anhui Province of China under Grant No. 2012cxy09.

\section{REFERENCES}

[1] Litovski V, Andrejević M, Zwolinski M. Analogue electronic circuit diagnosis based on ANNs [J]. Microelectronics Reliability, 2006, 46(8): 1382-1391.

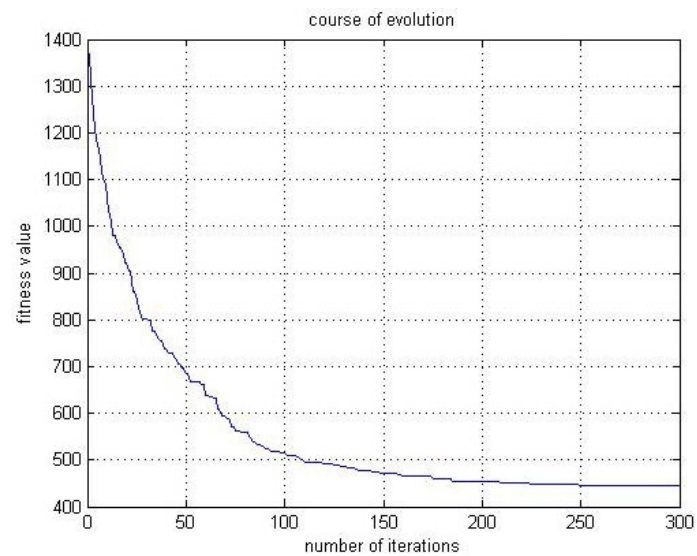

(a)
[2] Tingjun L, Zhongshan J, Xiuli Z, et al. Fault diagnosis of analog circuit based on multi-layer neural networks[C]//Electronic Measurement and Instruments, 2007. ICEMI'07. 8th International Conference on. IEEE, 2007: 3-331-3-334.

[3] Changbing Li, Maokang Du, Huiying Cao. Location strategy of logistics distribution centers based on hierarchical genetic algorithm [J]. Application Research of Computers, 2012, 29(1): 57-59.

[4] Liang Dong. Summary of logistics distribution center problem [J]. Science and Technology Information, 2013 (7): 148-148.

[5] Shishebori D, Mahnam M, Nookabadi A S. An efficient approach to discrete multiple different facility location problem[C]//Service Operations and Logistics, and Informatics, 2008. IEEE/SOLI 2008. IEEE International Conference on. IEEE, 2008, 2: 2519-2524.

[6] Fellows M R, Fernau H. Facility location problems: A parameterized view [J]. Discrete Applied Mathematics, 2011, 159(11): 1118-1130.

[7] Shang Gao, Jingyu Yang. Swarm intelligence algorithm and its application. China WaterPower Press,2006.

[8] Holland J H. Adaptation in natural and artificial systems: an introductory analysis with applications to biology, control, and artificial intelligence [J]. Cambridge: MIT Press, 1992.

[9] Yi S, Dezheng Z, Bin Z. A method of instance learning based on finitestate automaton and its application on TCM medical cases[C]//Computer, Mechatronics, Control and Electronic Engineering (CMCE), 2010 International Conference on. IEEE, 2010, 6: 427-430.

[10] Kilani M. Evolution and the complexity of finite automata [J]. International Game Theory Review, 2007, 9(04): 731-743.

[11] Berwick R C, Okanoya K, Beckers G J L, et al. Songs to syntax: the linguistics of birdsong [J]. Trends in cognitive sciences, 2011, 15(3): 113-121.

[12] Limin Ao, Shuda Wang, Yongzhi Guo. A developing model for software workflow based finite automaton[J]. Computer Engineering and Applications, 2004, 40(28): 208-211.

[13] Min Wan. The combination of automaton derivation and algorithm optimization [D]. Sichuan Normal University, 2004.

[14] Xiaoyuan Dong. Logistics center location method and its research [D]. University of International Business and Economics, 2006.

[15] L. Gao, Y. S. Ding, and H. Ying, An adaptive social network-inspired approach to resource discovery for the complex grid systems, International Journal of General Systems, 2006, 35(3): 347-360.

[16] L. Gao, Y.-S. Ding, and H. Ying, Economics-inspired decentralized control approach for adaptive grid services and applications, International Journal of Intelligent Systems, 2006, 21(12): 1269- 1288.

[17] L. Gao and A. Hailu, Comprehensive learning particle swarm optimizer for constrained mixed-variable optimization problems, International Journal of Computational Intelligence Systems, 2010, 3(6): 832-842.

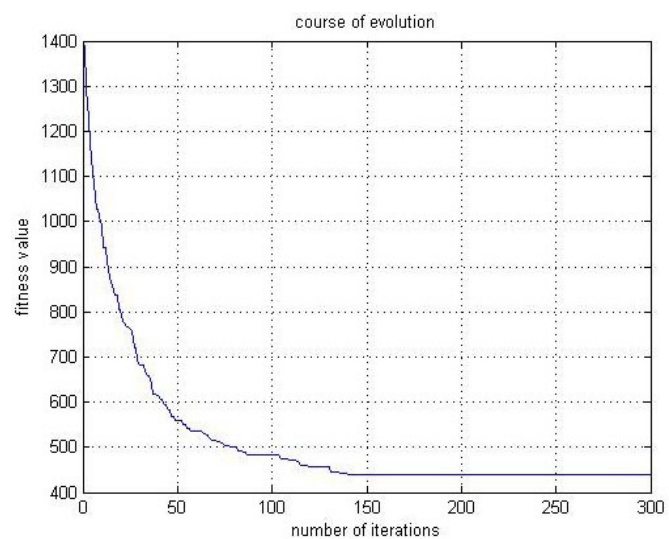

(b)

FIGURE II. THE EVOLUTIONS OF THE FITNESS FUNCTION: (a) THE EVOLUTION OF THE STANDARD GA, (b) THE EVOLUTION OF OUR PROPOSED ALGORITHM 